Information and Technology | Dr Mayank Kejriwal

\section{Crisis management:}

Using Artificial Intelligence to help save lives

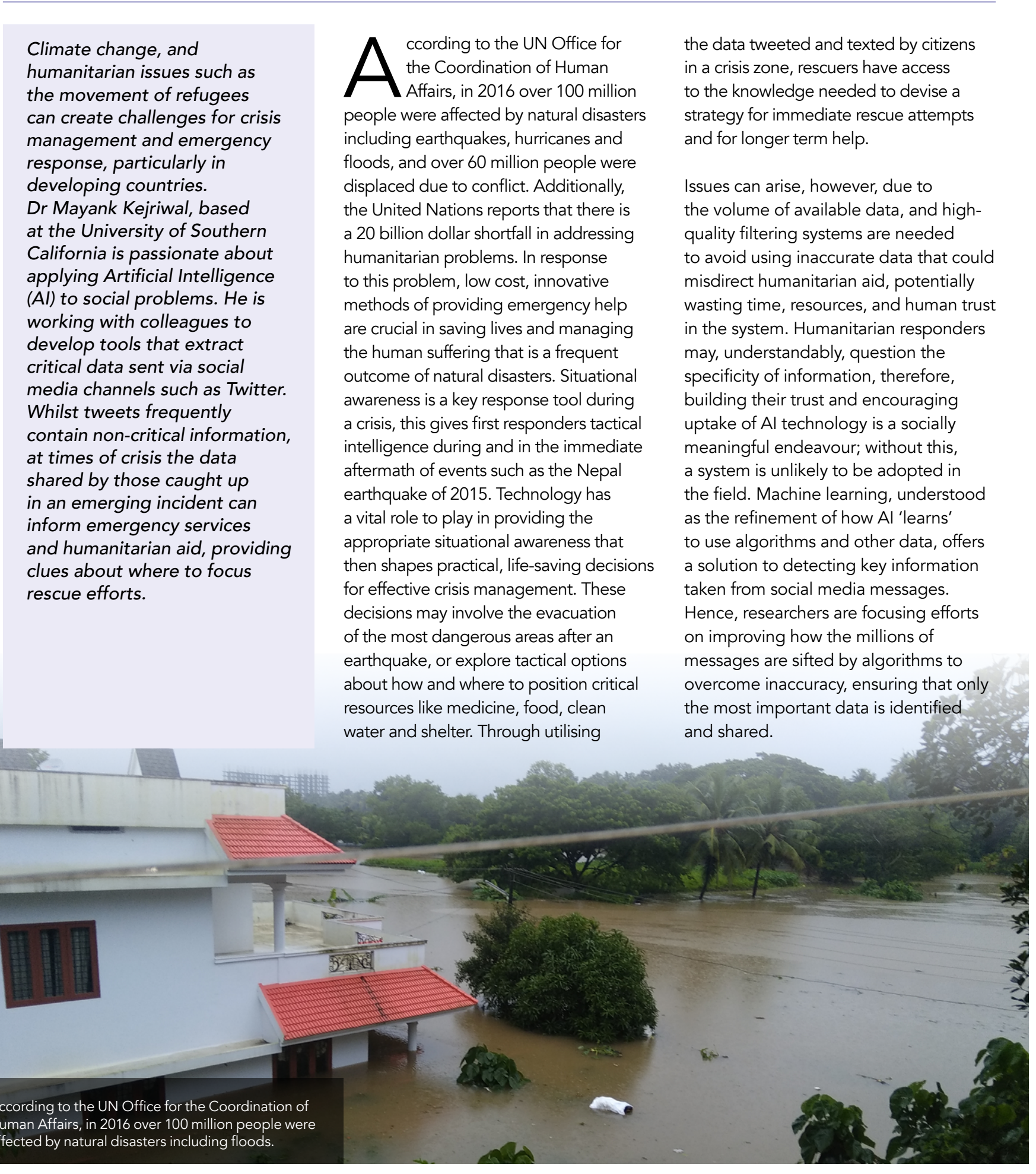

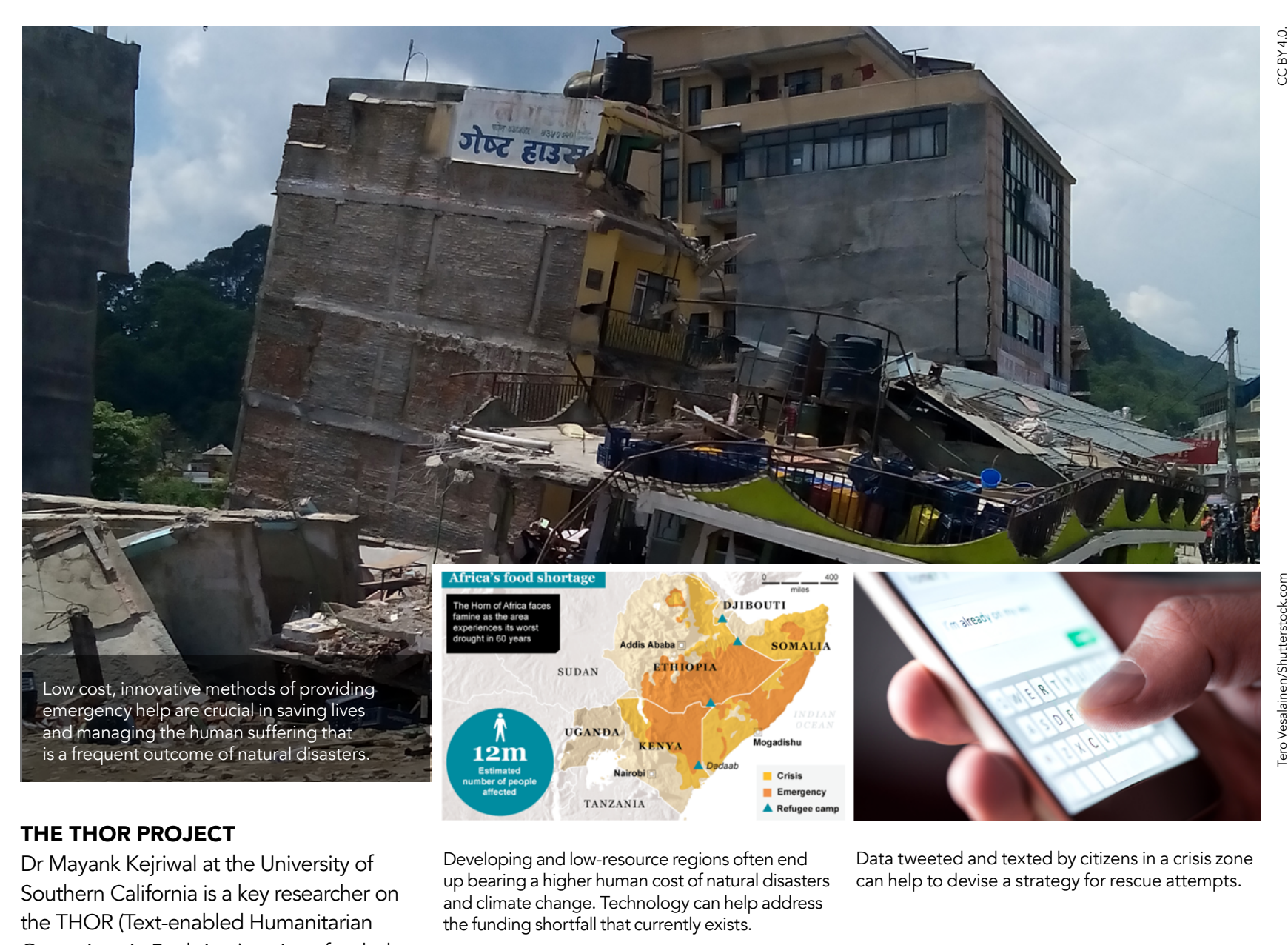

abled Humanitarian Onerations in Real-time) project, funded aim of the LAR's LORELEl program. The Langune LORELE (Low Resource project is to develop capabilities for low resource languages that are less common; human language technology is mostly designed for commonly spoken languages such as English. The focus of the project is to provide tactical insights language sent from commonty of personal devices such as mobile Vital data is extracted from messages sent on social media channels, with priority given to the most urgent intelligence that identifies key signals based on events, locations, topics and names. This realtime information helps first responders make rapid decisions such as moving people from buildings at risk of collapse. The system ensures that limited on-theground cognitive and physical resources are optimised to manage risk and danger. THOR is a LORELEl situation awarenes system that has been developed in collaborern wh reserch partners at Next Century Corporation andit
Technology has a vital role to play in providing the appropriate situational awareness that then shapes practical, life-saving decisions for effective crisis management.

data from social media, and from other externally developed Al systems, and data into English. Tweets and text messaging offers a ready supply interdisciplinary Al research is providing can detect and resolve urgent situation using this information. Twitter is one such social media platform that acts as an important channel for crisis informatics due to the avallability of the published data and its real-time application. During a humanitarian crisis many individuals with access to a phone will send messages relating to their current predicament, reporting on matters of Immediate concern to their well-being Some messages will be irrelevant, eve and individual's names are extracted of data information during a crisis, and (a keyword or subject label) the body of the message may lack data directly 列, or use idioms. Identifying and sorting through the 'corpus' or body of data in real-time is necessary for the rapid deployment of support. Clearly, this would be an impossible task for humans to complete without significant help, and this is where technological advances offer a neat solution. Key 'signals' or data relating to locations using algorithms that can then direct resources to casualties and responders. Importantly, these messages can be grouped according to urgency, with immediate need. 
SAVIZ: SITUATIONAL AWARENESS SAVIZ (situational awareness, visualisation, as an intgs, Crisis informatics), acts as an interactive visualisation plattorm that has been gathered together and sifted. SAVIZ has been designed to work with short, crisis-specific messages to ensure humanitarian aid is effectively deployed. Visualisation is an important component in a crisis informatics system helping responders make sense of large amounts of information. THO creates a user-friendly platform for situational awareness that can work with low-resource languages like Uighyur, a Turkic language, and Bengali which is primarily used in South Asia. Situationa awareness helps direct limited resources to the most important sites, translating messages when needed and providing the ground after a natural disaster. SAVIZ is a versatile platform, for example it is backward-compatible meaning that it can be used with pre-existing crisis intelligence platforms, and it can be utilised by non-technical first responders using an ordinary web browser. A brief demonstration of the system for potential users takes $1-3$ minutes, and an accompanying video illustrates the system's key features.

\section{LONG-LASTING SOCIAL IMPACTS}

As the system becomes further developed and embedded it has the potential for long-lasting social impact using readily avallable data sent from mobile phones
and similar devices, gathering information in a way that was not previously possible.

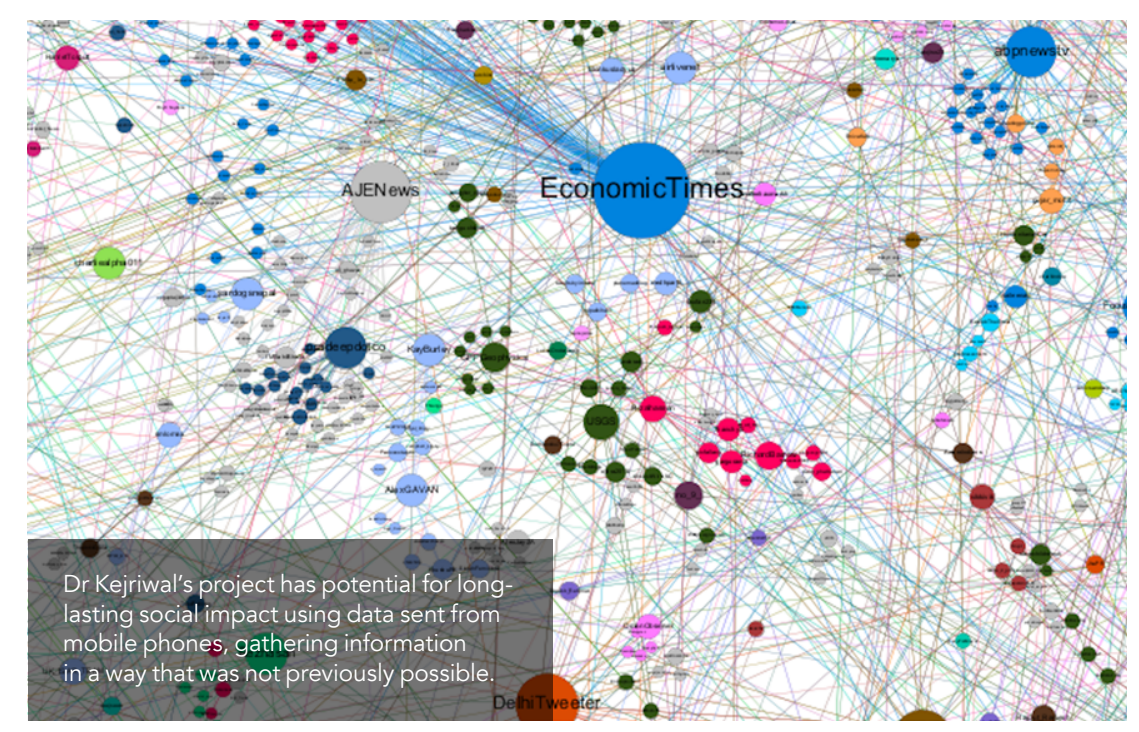

THOR: Text-enabled Humanitarian Operations in Real-time

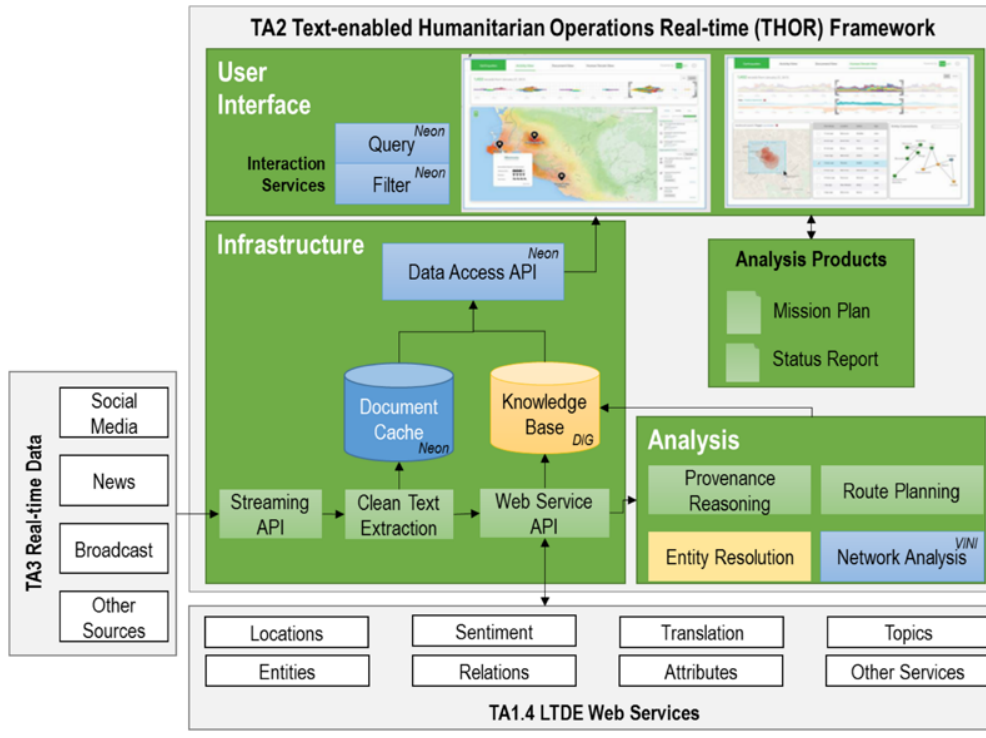

Figure 1: A schematic of THOR, which supports and integrates key Al modules, including natural
language processing and graph analytics. These modules were developed by many different group
over the lifetime of the $D A R P A L O R E L I$ program.

Freely available data sent over social media channels can help inform

humanitarian efforts, bringing help to those most in need before there is further damage or loss of life.

Dr Kejriwal and colleagues are also applying the technology to support law enforcement in the fight agains human trafficking. Whilst traffickers seek to hide data through tactics such as using emojis and misspellings in sex

through extracting information on phone numbers and locations. The use of timesensitive social media data can assist criss management during mass shootings, fres, large-scale accidents and epidemics such as the Ebola crisis, guiding assistance casualles and lagging the presence to diffuse conspiracy theories and malicious rumours, as was evidenced in the aftermath of the Las Vegas shootings of October 2017.

The work being done by Dr Kejriwal and colleagues offers rapid solutions to critica global concerns. Freely available data sent over social media channels can help inform humanitarian efforts, bringing help to those most in need before there is further damage or loss of life. As the research moves forward, new information networks and channels can be drawn on to further develop this lightweight, portable system for data sorting that in a position to provide immediate help.

\section{Behind the Research}

Dr Mayank Kejriwal

E: Kejriwal@isi.edu T: $\quad$ 1-217-819-6696 W: http://usc-isi-i2.github.io/kejriwal/

\section{Research Objectives}

Dr Kejiriwal's research deals with very challenging datasets covering a varier documents from both the Open and Dark Web, natural language documents, and social media documents like tweets. These datasets span challenging domains such as human trafficking, crisis informatics, causal reasoning and geopolitical forecasting.

\section{Detail}

4676 Admiralty Way, Ste. 1001

Marina del Rey, CA, Ste.

United States

Bio

Dr Mayank Kejriwal is research faculty at the University of Southern California, with a passion for applying Artificiad Intelligence to social problems such as human trafficking. His work has been featured in international academic and industrial venues. He is the author of an upcoming textbook on knowledge graphs (MIT Press).

\section{Funding}

Wratefully acknowledge the ongoing support and fur our partys. The views and conclusions containg herilod are those of the authors and should not be interpeted as necessarily representing the officil policies or endorsements, either expressed or implied, of DARPA, AFRL, or the U.S. Government.

\section{Collaborators}

We are extremely grateful to Next Century Corporation for engaging us in the THOR project. Next Century has been providing us with the data that we use in our research, and they have been largely responsible for engineering (and exploring the transition of) the Graphical User Interface that is used to showcase our research and algorithms.

\section{USCViterbi}

School of Engineering

\section{References}

Kejriwal M. \& Zhou P. (2019) SAVIZ: Interactive Exploration and Visualization of Situation Labeling Classifiers over Crisis Social Media Data, International Conference on Advances in
Social Networks Analysis and Mining, Vancouver, Aug 27-30, Social Networks Analysis and Mining, Vancouver, Aug 27-30, pp705-708.

Kejriwal M., \& Kapoor R. (2019) Network-theoretic information extraction quality assessment in the human trafficking doma s41109-019-0154-z

Kejriwal M., \& Gu Y. (2019) A Pipeline for Rapid PostCrisis Twitter Data Acquisition, Filtering and Visualization.

Technologies, 7(33). https://arxiv.org/pdf/1801.05881.pdf

\section{Personal Response}

What do you see as being the most important future these assist crisis management and humanitarian aid.

II Recenty, the have been many advincenentsin Natural Language Processing (NLP) induncements in Natural Language Processing (NLP), including question
and answering systems that can respond in an almost human-like fashion. However, these advancements have primarily been in English. With programs like DARPA LORELEl that funded my research, as well as several other groups, we are starting to see NLP-based A systems for non-English languages is obviously very important in the humanitarian domain, and these programs bode an exciting future for these tools. I think they will prove vital in helping
make our world a better place.

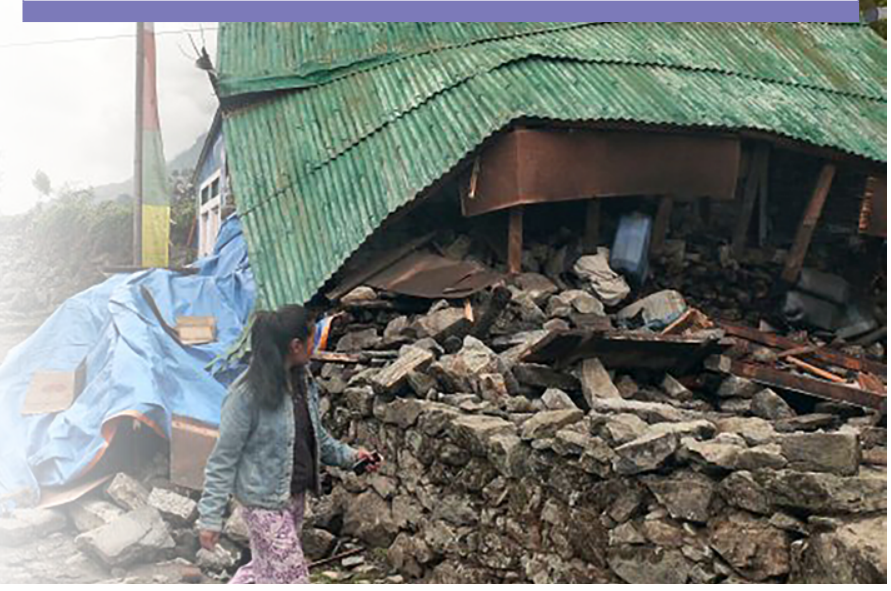

